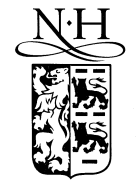

ELSEVIER

\title{
Graphical representation of non-absorbing polarization devices
}

\author{
Carlos R. Fernández-Pousa a , Ignacio Moreno ${ }^{\mathrm{a}, *}$, Noureddine Bennis ${ }^{\mathrm{b}}$, \\ Carlos Gómez-Reino ${ }^{\mathrm{c}}$, Carlos Ferreira ${ }^{\mathrm{b}}$ \\ a Departament de Ciència i Tecnologia de Materials, Universitat Miguel Hernández, Avda. Ferrocarril s / n, \\ E-03202 Elche (Alicante), Spain \\ ${ }^{\mathrm{b}}$ Departamento Interuniversitario de Optica, Universidad de Valencia, C / Dr. Moliner 50, E-46100 Burjassot, Spain \\ ${ }^{\mathrm{c}}$ Laboratorio de Optica. Departamento de Física Aplicada e Escola de Optica e Optometría. Universidade de Santiago de Compostela, \\ Campus Sur, E-15706 Santiago de Compostela, Spain
}

Received 7 March 2000; received in revised form 22 June 2000; accepted 18 July 2000

\begin{abstract}
A graphical representation of general non-absorbing polarization devices operating under normal plane-wave incidence is presented. The representation is based on a four-dimensional spherical parametrization of the Jones matrix of this kind of polarization devices. The graphical representation takes the form of a solid cylinder. The projection of the point representing the device over the base of the cylinder gives the corresponding polarization eigenvectors represented in the complex plane, while the height of the point in the cylinder is the phase of its eigenvalue. Some simple examples like wave-plates and rotators are discussed. The representation may represent a useful tool to identify the operation regimes of polarization modifying elements, for instance electro-optic devices. In this sense, the representation is also applied to the case of a twisted nematic liquid crystal display. (C) 2000 Elsevier Science B.V. All rights reserved.
\end{abstract}

Keywords: Polarization; Anisotropic media (crystal optics); Birefringence; Liquid-crystal devices; Polarization-sensitive devices; Spatial light modulators

\section{Introduction}

Polarization devices have been widely used as a tool for generation, analysis and modulation of totally polarized light [1-3]. Their mathematical description depends on the way that light polarization is described. The usual methods are by means of Jones vectors or Stokes parameters, the latter being capable to describe also partially polarized and unpo-

\footnotetext{
* Corresponding author. Tel.: +34-96-665-8409; fax: +34-96665-8793; e-mail: i.moreno@umh.es
}

larized light. In these two descriptions, polarization devices act as linear transformations, represented by Jones or Mueller matrices, respectively.

The complex plane is a useful graphical representation for completely polarized states of light. On the other hand, Poincaré sphere is a graphical representation of the light states through Stokes parameters. However, a graphical representation of the devices is lacking. In this paper we present a geometrical representation of polarization devices, based on Jones matrix formalism, which serves to identify non-absorbing polarization devices operating under normal 
plane-wave incidence. This definition includes simple devices like wave-plates and polarization rotators, and any combination of these elements. The graphical representation takes the form of a solid cylinder. It can be a useful tool to identify the operation mode when the device depends on a external parameter (typically an external voltage in electro-optic displays). For instance liquid crystal displays (LCD's) can be also represented in the cylinder since they may be regarded as a stack of anysotropic wave-plate layers [4,5]

The plan of this paper is the following. In Section 2 we describe a general four-dimensional Cartesian parametrization of the matrix representing the nonabsorbing polarization devices in Jones formalism, as well as some of its general properties. In Section 3, after a change to spherical variables, eigenvectors and eigenvalues of this matrix are presented. This is used to represent polarization devices in a solid cylinder in Section 4. Section 5 presents the loci in this cylinder corresponding to symmetry properties of reciprocal polarization devices previously analyzed in Ref. [6]. In Section 6 we address the representation of simple polarization devices, like waveplates, rotators and a combination of a centered wave-plate and a 90 degrees rotation. This last example represents the limit of operation of large birefringence for a 90-twist LCD [4,5]. This device is analyzed in detail in Section 7.

\section{Cartesian parametrization of non-absorbing polarization devices}

Assuming Jones formalism, outgoing light from a polarization device is represented by a $2 \times 1$ complex vector of the form $V_{\text {out }}=\left(E_{x}, E_{y}\right)^{t}$, where $E_{x}$ and $E_{y}$ are the complex components of the electric field in $x$ and $y$ directions in the laboratory frame, respectively, and $t$ means the transposed matrix. Propagation is assumed in the positive $z$ direction. If $V_{\text {in }}$ is the Jones vector representing the incoming light, both vectors are related by

$V_{\text {out }}=\mathbf{M}_{\mathrm{d}} \cdot V_{\text {in }}$

where $\mathbf{M}_{\mathrm{d}}$ is the $2 \times 2$ Jones matrix of the device. If we assume that this device does not absorb light, then $\mathbf{M}_{d}$ is a unitary matrix, i.e., it satisfies the condition $\mathbf{M}_{\mathrm{d}} \cdot \mathbf{M}_{\mathrm{d}}^{\dagger}=\mathbf{M}_{\mathrm{d}}^{\dagger} \cdot \mathbf{M}_{\mathrm{d}}=\mathbf{I}$ where the symbol $\dagger$ means the hermitic matrix, and $\mathbf{I}$ is the identity matrix [7]. In other words, $\mathbf{M}_{\mathrm{d}}$ belongs to the two-dimensional unitary group $\mathrm{U}(2)$. Therefore, its determinant is a pure phase, say $\operatorname{det}\left(\mathbf{M}_{\mathrm{d}}\right)=\exp (-2 i \beta)$, and then $\mathbf{M}_{\mathrm{d}}$ can be represented as [2-7]:

$$
\begin{aligned}
\mathbf{M}_{\mathrm{d}} & =\exp (-i \beta) \mathbf{M} \\
& =\exp (-i \beta)\left(\begin{array}{cc}
X-i Y & Z-i W \\
-Z-i W & X+i Y
\end{array}\right),
\end{aligned}
$$

in terms of the global phase shift of the device, $\beta$, and four real parameters $(X, Y, Z, W)$ such that $X^{2}+Y^{2}+Z^{2}+W^{2}=1$ so that $\operatorname{det}(\mathbf{M})=1$, i.e. $\mathbf{M}$ is an element of the two-dimensional special unitary group $\mathrm{SU}(2)$. This way of writing the matrix of Eq. (2), in terms of the parameters $(X, Y, Z, W)$ represents a generalization of a previous existing parametrization which has been applied to liquid crystal displays [8]. This representation uses three parameters $(X, Y, Z)$ with the restriction $X^{2}+Y^{2}$ $+Z^{2}=1$.

The overall phase in Eq. (2) is not of concern to us, since the polarization transformation capabilities of the device do not depend on $\beta$. Therefore, nonabsorbing polarization devices can be represented by elements of the group SU(2), parametrized by Cartesian coordinates $(X, Y, Z, W)$ in a three-dimensional sphere in four-dimensional Euclidean space. However, the general parametrization given by (2) is not unique, if we allow constant phases to be factored out. Indeed, if we assume a different decomposition of the same device as $\mathbf{M}_{\mathrm{d}}=\exp \left(-i \beta^{\prime}\right) \mathbf{M}^{\prime}$ with $\operatorname{det}\left(\mathbf{M}^{\prime}\right)=1$, then, since $\operatorname{det}\left(\mathbf{M}_{\mathrm{d}}\right)=\exp (-2 i \beta)$, it is necessary that $2 \beta^{\prime}=2 \beta+2 k \pi$, for $k$ any integer number. This means that the original parametrization of $\mathbf{M}$ is determined up to a minus sign:

$\mathbf{M}_{\mathrm{d}}=\exp (-i \beta) \mathbf{M}=\exp (-i \beta \pm i \pi)(-\mathbf{M})$.

If we restrict the study to the matrix $\mathbf{M}$, points ( $X$, $Y, Z, W)$ and $(-X,-Y,-Z,-W)$ parametrize matrices differing up to a constant phase $\exp ( \pm i \pi)$ and therefore, these points must represent the same device. This observation will be used in the following.

The Cartesian coordinates chosen to present the matrix $\mathbf{M}$ in Eq. (2) are essentially the Cayley-Klein 
or Euler parameters describing rotations in terms of $2 \times 2$ complex matrices [9]. Let us recall that any matrix $\mathbf{M}$ in $\mathbf{S U}(2)$ can be decomposed as:

$\mathbf{M}=e_{0} \mathbf{I}+i\left(e_{1} \sigma_{1}+e_{2} \sigma_{2}+e_{3} \sigma_{3}\right)$

where $\sigma_{1}, \sigma_{2}$, and $\sigma_{3}$ are the Pauli matrices:

$\sigma_{1}=\left(\begin{array}{ll}0 & 1 \\ 1 & 0\end{array}\right), \sigma_{2}=\left(\begin{array}{cc}0 & -i \\ i & 0\end{array}\right), \sigma_{3}=\left(\begin{array}{cc}1 & 0 \\ 0 & -1\end{array}\right)$

and $e_{0}, e_{1}, e_{2}$ and $e_{3}$ are real numbers named the Cayley-Klein or Euler parameters. Therefore, from Eq. (2), it is easy to identify $X=e_{0}, Y=-e_{3}$, $Z=e_{2}$ and $W=-e_{1}$. These four real parameters are known to describe a rotation in three-dimensional Euclidean space, up to an overall minus sign. This equivalence between three-dimensional rotations and polarization devices is easily visualized with the aid of the Poincare's sphere. If the device changes the state of polarization of light, but maintains the degree of polarization, the transformation induced in the polarization is visualized as a rotation in the Poincare sphere. This general transformation is described in Jones formalism, with the matrix $\mathbf{M}$ (see, for instance, the second proof of theorem I in Ref. [11] and references therein).

\section{Spherical parametrization of non-absorbing po- larization devices}

Since only three parameters of $(X, Y, Z, W)$ in Eq. (2) are independent, we seek for a three dimensional graphical representation of the device. An immediate idea consists in representing the device inside a three-dimensional solid sphere with a variable radius, for instance $1-W^{2}$. However, this representation does not give an easy identification of the eigenstates of the device (i.e., the incident polarization states that leave the device without change). The determination of the eigenstates of the device can be useful to optimize modulation properties of electrooptical displays $[10,11]$.

Thus, we use a more convenient representation based on four-dimensional spherical coordinates. This will allow to parametrize the $\mathbf{M}$ matrices in a form directly related to the Jones vector of the polarization eigenstates of the device, and represent them in a solid cylinder as it is described in the next section.

Since the four parameters $(X, Y, Z, W)$ vary in the range $[-1,+1]$ we can set:

$X=\cos \left(\phi_{1}\right)$

$Y=\sin \left(\phi_{1}\right) \cos \left(\phi_{2}\right)$

$Z=\sin \left(\phi_{1}\right) \sin \left(\phi_{2}\right) \cos \left(\phi_{3}\right)$

$W=\sin \left(\phi_{1}\right) \sin \left(\phi_{2}\right) \sin \left(\phi_{3}\right)$

where the angle $\phi_{3}$ belongs to the range $[0,2 \pi]$ while the angles $\phi_{1}$ and $\phi_{2}$ belong to the range $[0, \pi]$. The inverse relations are

$\phi_{1}=\cos ^{-1}(X)$

$\phi_{2}=\cos ^{-1}\left(\frac{Y}{\sqrt{1-X^{2}}}\right)$

$\phi_{3}=\arg (Z+i W)$

where arg stands for the argument of the complex number.

Therefore, with this notation, polarization devices are represented by three angles. Another parametrization of this kind was used in the early literature of the subject [12]. In that work, theorem I states that the matrix $\mathbf{M}$ of a general non-absorbing polarization device can be represented as a combination of two rotators of angles $\theta_{1}$ and $\theta_{2}$, represented by matrices $\mathbf{M}_{\mathrm{ROT}}\left(\theta_{1}\right)$ and $\mathbf{M}_{\mathrm{ROT}}\left(\theta_{2}\right)$, respectively, and a waveplate introducing a phase-shift $\delta$ which is centered in the laboratory frame, represented by the matrix $\mathbf{M}_{\mathrm{WP}}(\delta, 0)$, so that:

$\mathbf{M}=\mathbf{M}_{\mathrm{ROT}}\left(\theta_{1}\right) \cdot \mathbf{M}_{\mathrm{WP}}(\delta, 0) \cdot \mathbf{M}_{\mathrm{ROT}}\left(\theta_{2}\right)$.

This three-angle parametrization is different from the spherical parametrization presented here, which is adapted to the description of the polarization eigenstates and eigenvalues.

The calculation of the eigenvectors of a polarization device has been extensively published [2-4,11]. In the case of a non-absorbing polarization device, the eigenvectors of the matrix $\mathbf{M}$ can be calculated 
from Eq. (2). In terms of the spherical angles, the ratios between the complex amplitudes $E_{x}$ and $E_{y}$ of the eigenvectors $V^{ \pm}$take the following form [7]

$\zeta^{+}=\frac{E_{y}^{+}}{E_{x}^{+}}=\frac{1}{\tan \left(\frac{1}{2} \phi_{2}\right)} \exp \left[i\left(\phi_{3}+\frac{\pi}{2}\right)\right]$

$\zeta^{-}=\frac{E_{y}^{-}}{E_{x}^{-}}=\tan \left(\frac{1}{2} \phi_{2}\right) \exp \left[i\left(\phi_{3}-\frac{\pi}{2}\right)\right]$

and their corresponding eigenvalues $\lambda^{ \pm}$are

$\lambda^{ \pm}=\exp \left( \pm i \phi_{1}\right)$

These equations show that the eigenvectors of the device depend only on the angles $\phi_{2}$ and $\phi_{3}$, while the eigenvalues are phase-only values (as expected for a non-absorbing device) with phase equal to $\pm \phi_{1}$. Notice that the above indetermination associated to the constant phase $\exp ( \pm i \pi)$ results only in an indetermination of the sign of the eigenvalues, $\pm \lambda^{ \pm}$. We shall refer to the positive (negative) eigenvector to that one associated to the positive (negative) value $\phi_{1}$ in exponent of Eq. (10).

Once the eigenvalues and eigenvectors are known, the original matrix $\mathbf{M}$ can be recovered by a simple change of basis:

$\mathbf{M}(X, Y, Z, W)=\mathbf{U}^{\dagger}\left(\phi_{2}, \phi_{3}\right) \cdot \mathbf{D}\left(\phi_{1}\right) \cdot \mathbf{U}\left(\phi_{2}, \phi_{3}\right)$

where

$\mathbf{D}\left(\phi_{1}\right)= \pm\left(\begin{array}{cc}\exp \left(i \phi_{1}\right) & 0 \\ 0 & \exp \left(-i \phi_{1}\right.\end{array}\right)$

and

$$
\begin{aligned}
& \mathbf{U}\left(\phi_{2}, \phi_{3}\right) \\
& =\left(\begin{array}{cc}
\sin \left(\frac{\phi_{2}}{2}\right) & -i \cos \left(\frac{\phi_{2}}{2}\right) \exp \left(-i \phi_{3}\right) \\
-i \cos \left(\frac{\phi_{2}}{2}\right) \exp \left(+i \phi_{3}\right) & \sin \left(\frac{\phi_{2}}{2}\right)
\end{array}\right)
\end{aligned}
$$

\section{Solid cylinder representation}

The complex plane is a useful graphical representation for completely polarized light, directly related to the Jones vectors [1]. The state of polarization is characterized by a complex number equal to the ratio between the complex amplitudes of the Jones vector $\zeta=E_{y} / E_{x}$. Points lying in the pure imaginary axis correspond to elliptical states where the major and minor axes of the ellipse are parallel to the coordinate system axes. Points lying in the pure real axis correspond to linearly polarized light. In particular the origin of the complex plane represents linear polarized light oriented in $x$ direction, while the infinity corresponds to linear polarized light oriented in $y$ direction. Finally, those points located in the unitary radius circle are polarized states where the amplitudes in $x$ and $y$ direction have the same modulus.

The representation of the two eigenstates (Eqs. (9a) and (9b)) in the complex plane is shown in Fig. 1. Because they are two orthogonal states they lie in opposite directions respect to the origin of the complex plane. One modulus is the inverse of the other, being equal to $1 / \tan \left(\phi_{2} / 2\right)$ for the case with eigenvalue $\lambda^{+}=\exp \left(+i \phi_{1}\right)$ while being $\tan \left(\phi_{2} / 2\right)$ for the case with eigenvalue $\lambda^{-}=\exp \left(-i \phi_{1}\right)$. Let us note that always one of the two eigenvectors lie inside the circle of unity radius. In the limit case where $1 / \tan \left(\phi_{2} / 2\right)=\tan \left(\phi_{2} / 2\right)= \pm 1$ both states are in the border of the circle.

The proposed graphical representation for non-absorbing polarization devices consists in a three-dimensional representation in the form of a solid cylin-

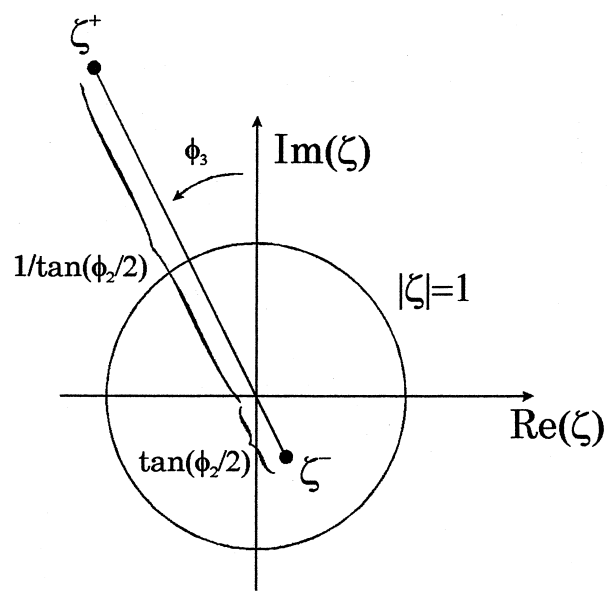

Fig. 1. Complex representation of the two polarization eigenstates of a non-absorbing device as a function of the two spherical angles $\phi_{2}$ and $\phi_{3}$. 
der with unit radius and height $\pi$. This cylinder is constructed as follows, see Fig. 2a. The base of the cylinder rests on the complex $\zeta$ plane, that allows to represent both eigenvectors, $\zeta^{ \pm}$. Above and below these two points we represent, as a third coordinate, the phase of the corresponding eigenvalues. The indetermination in the sign of $\mathbf{M}$ results in two possibilities for each eigenvector. Associated to the eigenvector $\zeta^{+}$we represent the values of $+\phi_{1}$ and $+\phi_{1}-\pi$, and associated to $\zeta^{-}$the values $-\phi_{1}$ and $-\phi_{1}+\pi$. These values are chosen in order to be in the range $[-\pi, \pi]$. Then, it is straightforward to notice that there exists at least one point in the solid cylinder with unit radius and height $\pi$. This single point in this cylinder contains the information
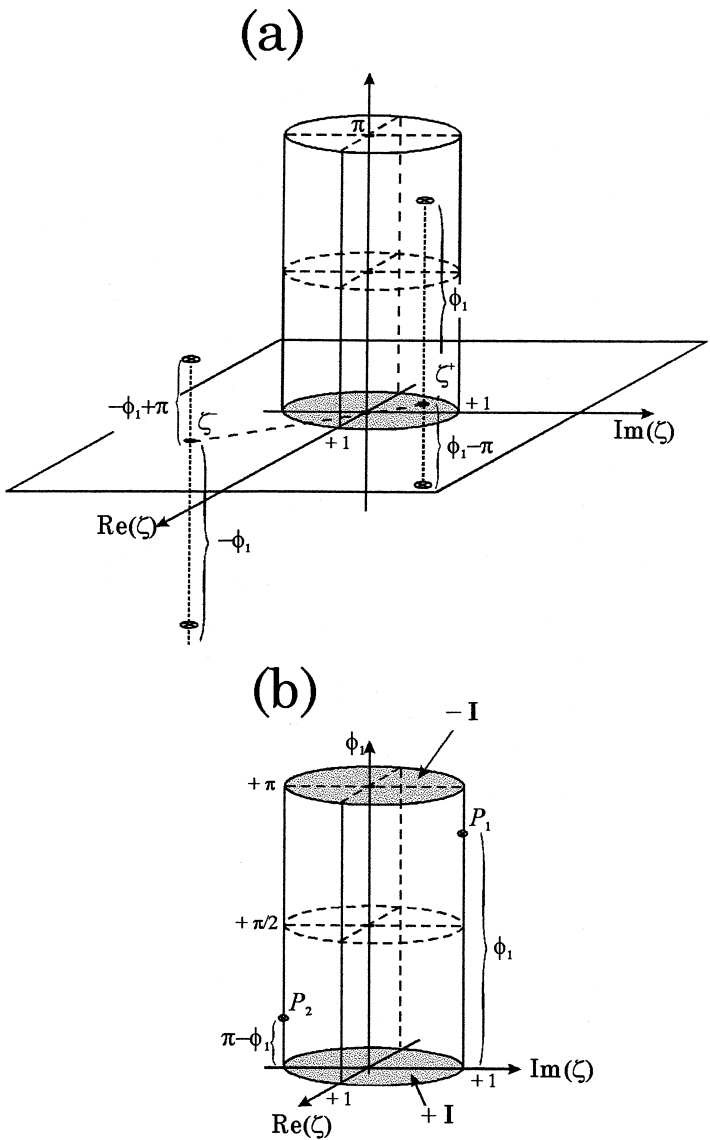

Fig. 2. Cylindrical representation for a non-absorbing polarization device. (a) Relation with eigenvalues and eigenvectors. (b) Resulting solid cylinder and identifications. necessary to reconstruct the Jones matrix for the device, up to a global phase. The projection over the base of the cylinder yields one eigenvector, so that it is a direct task to compute the orthogonal state, which is the other eigenvector of the device. Up to a global minus sign, the eigenvalue of the first eigenvector is dictated by the height of the representative point. The eigenvalue of the second eigenvector is the complex conjugated phase. This procedure yields one of the two possibilities for the matrix $\mathbf{M}$, which are equivalent up to a global phase shift (see Eq. (3)). Fig. $2 b$ shows the scheme of the resulting representation.

Some identifications of the representative points occur in the borders of the cylinder. In the limit case when both eigenvectors lie in the unitary circle, two points in the surface of the cylinder represent the same device, corresponding to heights $\phi_{1}$ and $\pi-$ $\phi_{1}$. For instance, points $P_{1}$ and $P_{2}$ in Fig. $2 \mathrm{~b}$ represent the same device. Moreover, all the points in the base of the cylinder and in the plane $\phi_{1}=\pi$ are equivalent, since they represent $\mathbf{I}$ and $-\mathbf{I}$, respectively. No identifications occur inside the solid cylinder, so different points represent different devices.

Notice that the proposed representation identifies directly the form of the eigenstates in the complex plane, which is a natural way of representation of polarization states when they are expressed in Jones matrix formalism.

\section{Loci associated to symmetry properties of re- ciprocal devices}

Additional properties can be discussed if we assume that $\mathbf{M}_{\mathrm{d}}$ represents a reciprocal device. In Ref. [2] Brosseau defines a reciprocal device as an optical system which satisfies the principle of reciprocity. It states that the input and output of any linear passive optical system can be interchanged without altering the response of the optical system. Optical reciprocity can be applied to obtain some general properties of the Jones matrix [13]. In order to do that, it is necessary to provide a relation between the action of the device under direct and backward illumination. 
Let $\tilde{\mathbf{M}}_{\mathrm{d}}$ denote the matrix for the device under backward illumination. It is related to $\mathbf{M}_{\mathrm{d}}$ by [6]:

$\tilde{\mathbf{M}}_{\mathrm{d}}=\sigma_{3} \cdot \mathbf{M}_{\mathrm{d}}^{t} \cdot \sigma_{3}$

In this equation, both $\mathbf{M}_{\mathrm{d}}$ and $\tilde{\mathbf{M}}_{\mathrm{d}}$ describe the action of the device when incoming light is expressed in two different right-handed reference frames which correspond to forward and backward propagation, respectively. The use of two reference frames requires the introduction of $\sigma_{3}$ in Eq. (13) to implement the relative inversion of axes (for an application to reflective polarization systems see Ref. [14]). The comparison of the action of the device under forward or backward illumination, together with relation (13), can be used to deduce the cancellation of some of the parameters $(X, Y, Z, W)[6]$, a fact that reflects a symmetry property of the display. The cancellation of one of these parameters defines particular regions on the cylinder, and they can be summaryzed as follows:

1. If the response of the device under inverse illumination is exactly equal to the response under direct illumination, then $W=0$ and consequently $\phi_{3}=0$ if $Z>0$, or $\phi_{3}=\pi$ if $Z<0$. These devices are represented in the cylinder by the plane $\operatorname{Re}(\zeta)=$ 0 and the corresponding eigenvectors are always ellipses with axes centered on the coordinate axis.

2 . If the response of the device under inverse illumination is equal to the response under direct illumination, up to inversion in one of the two transverse axes, then $Z=0$ and consequently $\phi_{3}=$ $\pi / 2$ if $W>0$, or $\phi_{3}=3 \pi / 2$ if $W<0$. In this case the devices are represented in the plane $\operatorname{Im}(\zeta)=0$ and the eigenvectors are always linearly polarized states.

3. If the response of the device under inverse illumination is equal to the response under direct illumination, up to a rotation of \pm 90 degrees, then $Y=0$. Consequently, $\phi_{2}=\pi / 2$ and thus $\left|\zeta^{ \pm}\right|=1$, so that the devices lie in the surface of the cylinder. The eigenstates have the same modulus in $x$ and $y$ directions.

Fig. 3a-Fig. 3c show the representation in the cylinder of the regions corresponding to symmetry properties 1, 2 and 3 respectively.

\section{Examples}

In this section we analyze some well known polarization devices and their representation in the cylinder.

\subsection{Rotators}

As a first example, we will consider a rotator of angle $\theta$, whose Jones matrix is:

$\mathbf{M}_{\mathrm{ROT}}(\theta)=\left(\begin{array}{cc}\cos \theta & -\sin \theta \\ \sin \theta & \cos \theta\end{array}\right)$.

This is an example of reciprocal device that verifies property 1 . In this case, the parameters for the representation are $\phi_{1}=\theta,\left|\zeta^{ \pm}\right|=1$ and $\phi_{3}=0$ if $\sin \theta<0$, or $\phi_{3}=\pi$ if $\sin \theta>0$. Fig. 4 shows the (a)

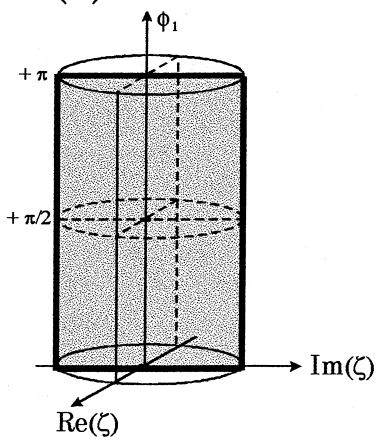

(b)

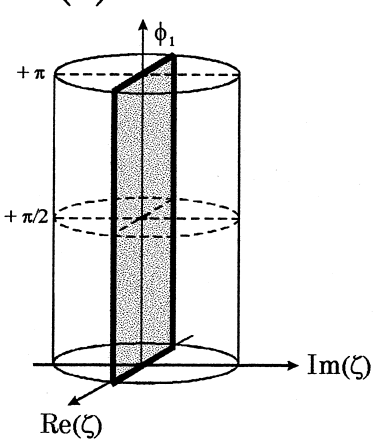

(c)

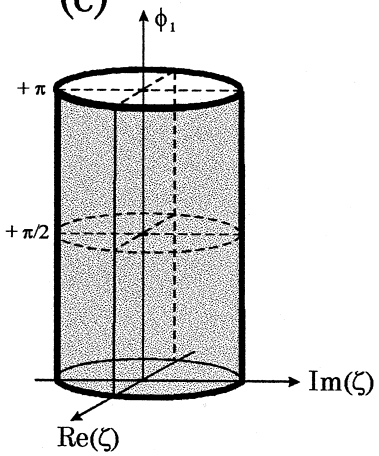

Fig. 3. (a) Devices verifying symmetry property 1, (b) Devices verifying symmetry property 2, (c) Devices verifying symmetry property 3. 


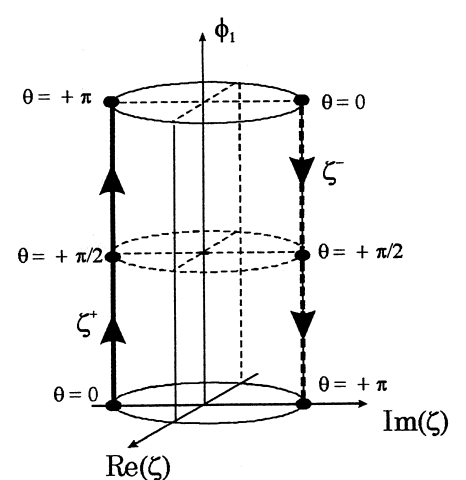

Fig. 4. Cylinder representing rotators of angle $\theta$, varying from 0 to $\pi$.

points in the cylinder representing a rotator characterized by a rotation angle $\theta$, taking values from $\theta=0$ up to $\theta=\pi$. Notice that, since we have allowed to factor out a minus sign, a rotation in the range $[\pi, 2 \pi]$ is equivalent to another rotation in the range $[0, \pi]$. As expected, the projection over the complex plane give the $L$ - and $R$-circular polarized eigenstates. Because the points lie in the border of the cylinder, two points represent the device. The continuous line in Fig. 4 corresponds to the eigenvalue with positive phase, while the dotted line corresponds to the one with negative phase.

\subsection{Waveplates}

The second example is a wave-plate that introduces a phase-shift $\delta$ which is rotated an angle $\theta$ with respect to the laboratory coordinate axis, $\mathbf{M}_{\mathrm{WP}}(\delta, \theta)$. The corresponding Jones matrix is [1]:

$$
=\left(\begin{array}{cc}
\cos \left(\frac{\delta}{2}\right)+i \sin \left(\frac{\delta}{2}\right) \cos (2 \theta) & i \sin \left(\frac{\delta}{2}\right) \sin (2 \theta) \\
i \sin \left(\frac{\delta}{2}\right) \sin (2 \theta) & \cos \left(\frac{\delta}{2}\right)-i \sin \left(\frac{\delta}{2}\right) \cos (2 \theta)
\end{array}\right) .
$$

This is a reciprocal revice verifying property 2 . In this case $\phi_{1}=\delta / 2,\left|\zeta^{-}\right|=|\tan (\theta)|,\left|\zeta^{+}\right|=1 /|\tan (\theta)|$ and $\phi_{3}=\pi / 2$ if $\sin (\delta / 2) \sin (2 \theta)<0$, or $\phi_{3}=$ $3 \pi / 2$ if $\sin (\delta / 2) \sin (2 \theta)>0$. The points representing the device lie always in the plane $\operatorname{Im}(\zeta)=0$ and consequently the eigenvectors are linearly polarized, corresponding to the directions of the neutral axes of the waveplate. The height over the base of the cylinder gives half the phase-shift $(\delta / 2)$ and the distance from the center depends only on the angle $\theta$. In Fig. 5a and Fig. 5b we analyze the cases $\theta=0$ and $\theta=\pi / 4$, respectively, when the phase of the wave-plate changes from 0 to $2 \pi$. In the first case (Fig. 5a), the device follows the axis of the cylinder as the phase-shift increases. The projection gives always the origin of the complex plane, corresponding to a horizontal linearly polarized light. The second eigenvector is at infinity, corresponding to the vertical linearly polarized light. Fig. $5 \mathrm{~b}$ shows the circuit for $\theta=\pi / 4$. In this case the device follows the lines in the surface corresponding to $\operatorname{Re}(\zeta)=$ \pm 1 . The projection on the complex plane gives the
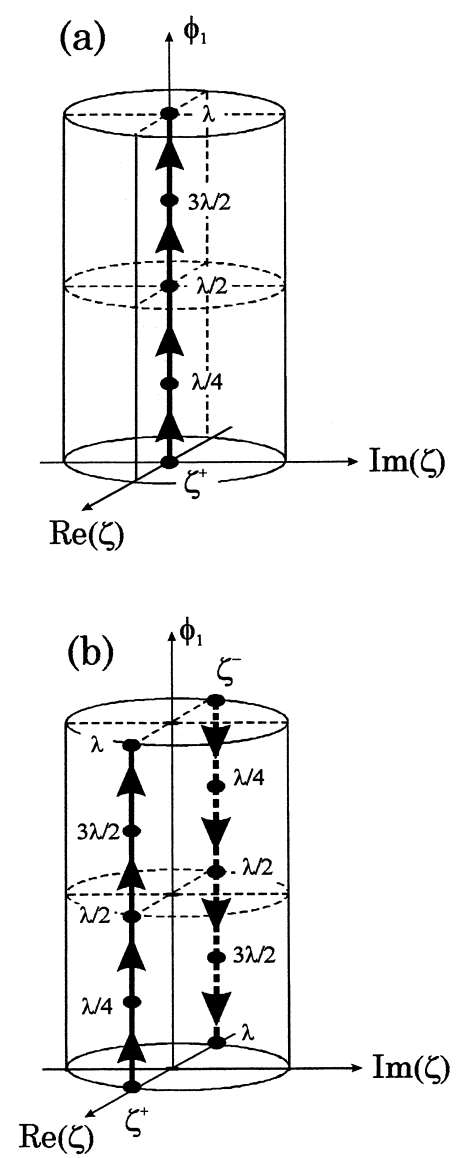

Fig. 5. Cylinder representing wave-plates with phase shift $\delta$ varying form 0 to $2 \pi$. (a) Wave-plates centered in reference axes. (b) Wave-plates rotated an angle $\theta=\pi / 4$. The $\lambda$ 's refer to the standard notation of wave-plates. 
two linearly polarized states oriented at $\pm(\pi / 4)$. Again two lines are present in the graphic representing the eigenvalues with positive phase (continuous line) and negative phase (dotted line).

\subsection{Waveplate plus a rotator}

A third example consists in the combination of a centered wave-plate and a rotation of 90 degrees. It is given by the following matrix product

$$
\begin{aligned}
\mathbf{M}_{\mathrm{WP}+\mathrm{ROT}} & =\mathbf{M}_{\mathrm{ROT}}\left(\frac{\pi}{2}\right) \cdot \mathbf{M}_{\mathrm{WP}}(\delta, 0) \\
& =\left(\begin{array}{cc}
0 & -\exp \left(-i \frac{\delta}{2}\right) \\
+\exp \left(+i \frac{\delta}{2}\right) & 0
\end{array}\right) .
\end{aligned}
$$

In this case $\phi_{1}=\pi / 2,\left|\zeta^{ \pm}\right|=1$ and $\phi_{3}=(\pi / 2) \pm$ $\pi \epsilon[0,2 \pi]$. Fig. 6 shows the evolution of the device as a function of $\delta$ in the range from 0 to $2 \pi$. It follows the border of the cylinder at height $\phi_{1}=$ $\pi / 2$. The projection over the complex plane gives eigenstates which are always centered ellipses which respect to the coordinate axes. This combination is interesting because it represents the limit for a 90 degrees twisted nematic liquid crystal structure, when the device is in the adiabatic following approximation (or waveguiding limit) [15].

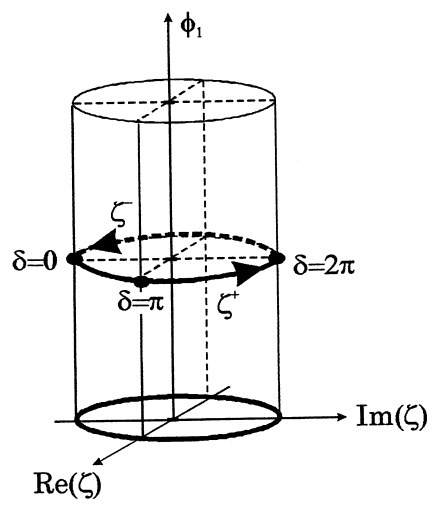

Fig. 6. Cylinder representing the combination of a wave-plate with phase shift $\delta$ varying form 0 to $2 \pi$, and a rotator of 90 degrees.

\section{Representation of the operation regimes of a 90-twist LCD}

We will apply this graphical representation to a twisted anysotropic media. It consists on an anysotropic media which neutral axes describe a 90 degrees twist from the incident to the back surface of the crystal. Yariv and Yeh analyzed this structure in ref. [4]. This type of model has also been used in the description of a 90-twist LCD [5,15]. The Jones matrix of this device, assuming that the ordinary and extraordinary axes at the input surface are oriented parallel to the laboratory axes, is given by:

$$
\begin{aligned}
& \mathbf{M}_{90 \text { Twist }}(\beta)=\exp (-i \beta) \\
& \times\left(\begin{array}{cc}
\frac{\pi}{2 \gamma} \sin (\gamma) & \cos (\gamma)+i \frac{\beta}{\gamma} \sin (\gamma) \\
-\cos (\gamma)+i \frac{\beta}{\gamma} \sin (\gamma) & \frac{\pi}{2 \gamma} \sin (\gamma)
\end{array}\right)
\end{aligned}
$$

where $\beta \geq 0$ is the birefringence of the display, and $\gamma=\sqrt{\beta^{2}+(\pi / 2)^{2}}$. Thus, it is obtained

$\phi_{1}=\cos ^{-1}\left(\frac{\pi}{2 \gamma} \sin (\gamma)\right)=\cos ^{-1}\left(\frac{\pi}{2} \operatorname{sinc}\left(\frac{\gamma}{\pi}\right)\right)$

$\phi_{3}=\arg \left(\cos (\gamma)-i \frac{\beta}{\gamma} \sin (\gamma)\right)$

and $\left|\zeta^{ \pm}\right|=1$. Their propagation eigenstates are elliptic polarized states, where the modulus of the components of the electric field is equal for $x$ and $y$ directions.

The representation of these devices for a range of values $\beta \geq 0$ is shown in Fig. 7, where only the eigenvector with positive phase has been drawn for clarity. The circuit lies in the border of the cylinder, as it is expected owing to the form of its propagation eigenstates [11].

This representation may be useful to describe the operation modes of the device. For $\beta \rightarrow 0$ the representative point shows that the eigenvectors tend to be linear states oriented at $\pm 45^{\circ}$ as it is easy to show by exploring the $\beta \rightarrow 0$ limit in $\phi_{3}$. As $\beta$ (or $\gamma$ ) increases, the values of the phase $\phi_{1}$ are given by the composition of the decreasing function $\cos ^{-1}$ 


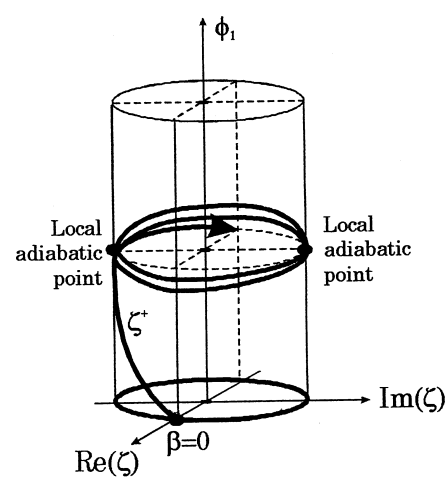

Fig. 7. Cylinder representing a 90-degrees twisted anysotropic media with birefringence $\beta \geq 0$. Only the positive eigenvector-eigenvalue is represented.

with the oscillating sinc function (see Eq. (18a)). The eigenvectors are no longer linear, and the phase of the eigenvalues oscillate around the value $\phi_{1}=$ $(\pi / 2)$. Maximum and minimum values of $\phi_{1}$ are reached whenever $\gamma=\tan \gamma$, corresponding to the minimum and maximum values of the sinc function, respectively

For large values of $\beta$ (or $\gamma$ ) the device tends to be located along the plane $\phi_{1}=(\pi / 2)$, since the sinc function tends to zero. In this situation, the operation of the device tends to be equivalent to the last example, the combination of a wave-plate and a rotation of 90 degrees. This operation regime was named by Yariv and Yeh as the adiabatic following regime of the display [4].

Points verifying $\gamma=k \pi$, where $k$ is any positive integer, correspond to zeroes of the sinc function, and they occur always between maximum and minimum values of this function. They give points located at $\phi_{3}=0$ or $\phi_{3}=\pi$, so that the device operates as a 90 degrees rotator (see Fig. 4). Their representative points lie exactly in the plane $\phi_{1}=$ $(\pi / 2)$. These points have been named as local adiabatic points, in analogy with the previous terminology of LCD's, and they have been used for the determination of the optical physical parameters of the display [16].

\section{Conclusions}

A graphical representation for non-absorbing polarization devices has been presented. It takes the form of a solid cylinder with the base of radius equal to one, and a maximum height of $\pi$. The projection of the point representing the device over the base directly gives one of the eigenvectors of the Jones matrix represented in the complex plane. Several examples of well known devices have been presented. It has also been applied to a twisted nematic liquid crystal display. This is an example of electrooptical device, where the polarization properties depend on an external parameter, typically an applied voltage. For these electro-optical devices, the geometrical representation may be used to visualize the operation regimes of the display.

\section{Acknowledgements}

Ignacio Moreno and Carlos Ferreira acknowledge financial support from Dirección General de Enseñanza Superior del Ministerio de Educación y Cultura from Spain, project number PB96-1134-C0202. The work of Carlos Gómez-Reino is supported partially by Xunta de Galicia, Spain, under contract PGIDT99PXI22201B.

\section{References}

[1] S. Huard, Polarisation de la lumière, Masson, Paris, 1994.

[2] R.M.A. Azzam, N.M. Bashara, Ellipsometry and Polarized Light, Elsevier, Amsterdam, 1980.

[3] C. Brosseau, Fundamentals of Polarized Light, John Wiley and Sons, New York, 1998.

[4] A. Yariv, P. Yeh, Optical Waves in Crystals, John Wiley and Sons, New York, 1984.

[5] K. Lu, B.E.A. Saleh, Theory and design of the liquid crystal TV as an optical spatial phase modulator, Opt. Eng. 29 (1990) 240-246.

[6] C.R. Fernández-Pousa, I. Moreno, N. Bennis, C. Gómez-Reino, Generalized formulation and symmetry properties of reciprocal non-absorbing polarization devices, Application to liquid crystal displays, J. Opt. Soc. Am. A, to be published.

[7] R.C. Jones, New calculus for the treatment of optical systems. I. Description and discussion of the calculus, J. Opt. Soc. Am. 31 (1941) 488-493.

[8] J.L. Bougrenet de la Tocnaye, L. Dupont, Complex amplitude modulation by use of liquid-crystal spatial light modulators, Applied Optics 36 (1997) 1730-1741.

[9] H. Goldstein, Classical Mechanics, 2nd edn., Addison-Wesley, Reading, 1987. 
[10] J.L. Pezzaniti, R.A. Chipman, Phase-only modulation of a twisted nematic liquid-crystal TV by use of the eigenpolarization states, Optics Letters 15 (1993) 1567-1569.

[11] J.A. Davis, I. Moreno, P. Tsai, Polarization eigenstates for twisted nematic liquid crystal displays, Applied Optics 37 (1998) 937-945.

[12] H. Hurwitz, R.C. Jones, New calculus for the treatment of optical systems. II Proof of three equivalence theorems, J. Opt. Soc. Am. 31 (1941) 493-499.

[13] N. Vansteenkiste, P. Vignolo, A. Aspect, Optical reversibility theorems for polarization: application to remote control of polarization, J. Opt. Soc. Am. A 10 (1993) 2240-2245.

[14] J. Goodman, Introduction to Fourier Optics, 2nd edn., McGraw-Hill, New York, 1996.

[15] P. Yeh, C. Gu, Optics of Liquid Crystal Displays, John Wiley and Sons, New York, 1999.

[16] I. Moreno, N. Bennis, J.A. Davis, C. Ferreira, Twist angle determination in liquid crystal displays by location of local adiabatic points, Opt. Commun. 158 (1998) 231-238. 